\title{
Wearable Sensor Control of Above-Knee Prosthetic Device
}

\section{Zlata Jelačić*}

Assistant Professor, Department of Mechanics, University of Sarajevo, Bosnia and Herzegovina

*Corresponding Author: Zlata Jelačič, Assistant Professor, Department of Mechanics, Faculty of Mechanical Engineering, University of Sarajevo, Bosnia and Herzegovina.

Received: May 07, 2019; Published: June 03, 2019

DOI: $10.31080 /$ ASOR.2019.02.0060

\begin{abstract}
Most amputees use passive prostheses, meaning that these devices are not powered in any way and cannot give any additional output of energy to its user. They usually work on using elastic or some other potential energy which is stored during one sequence of gait, and then released in another. However, performing high power demanding tasks, such as stair ascent, presents a problem, because the lack of muscles makes it impossible to produce the required forces. To perform high demanding power activities, prosthesis must be powered, primarily in its main joints - knee and ankle. Review of the literature posed important open questions regarding sensorimotor interaction during human-human interactions that could facilitate the design of human-robot interactions and haptic interfaces for prosthetic design. The goal is to use the leading principles of the human-human interaction in order to define a way in which people could be in contact with robots in a more intuitive and biologically inspired way. In this paper, we present the wearable sensor real time control of newly developed above-knee prosthetic SmartLeg prototype with powered knee and ankle joints. Specialized control unit is developed in order to achieve required kinematics and dynamics to enable it to perform high power demanding activities in a more natural manner, especially stair ascend.
\end{abstract}

Keywords: Powered (Active) Lower-Limb Prostheses; Wearable Sensors; Real Time Control; Rehabilitation Robotics; Human-Machine Interface

\section{Abbreviations \\ RPBC: Robust Passivity Based Control.}

\section{Introduction}

Recently there has been a significant interest, both in academia and industry, in devising advanced solutions for the improvement of mobility of people with lower limb amputation. Scientific and technical innovations have made a progress towards developing more comfortable, efficient and lifelike artificial limbs. Future progress is likely to depend on the interaction between three powerful forces: amputee's demands, advances in surgery and engineering, and sufficient healthcare funding to sustain development and application of technological solutions. Prosthetic technology has advanced to a remarkable degree in the past two decades, driven largely by amputees' demand. Today, otherwise healthy individuals with transfemoral amputation should be able to participate in a full range of normal activities, to walk without any perceptible limp, and to engage in recreational and sports activities [1].

In developed countries the main cause of lower limb amputation is circulatory dysfunction. The prime reason for this is atherosclerosis; although up to a third of patients have concomitant diabetes. These people are usually in their sixth decade (or older), and most have additional health problems that limit their walking ability [2]. This is in sharp contrast with developing countries, where most amputations are caused by trauma related either to conflict or to industrial or traffic injuries [3]. The devastation caused by land mines continues, particularly when displaced civilian return to mined areas and resume agricultural activities [4]. Global extrapolations are problematic, but a recent US study states that the amputation rate among combatants in recent US military conflict remains at $14-19 \%$ [5]. 
The single most critical aspect of any prosthesis is the quality of the interface between the limb remnants (stump) and the artificial prosthesis. The portion of the prosthesis that fits snugly over the limb remnant, the socket, determines the amputee's comfort and ability to control the artificial limb. Since the 1980s prosthetic clinicians and researches worldwide have made breakthroughs in design and materials that have greatly improved the connection between the socket and stump. Currently, silicone elastomers are widely used to create a soft and slightly elastic inner liner, providing a thin, comfortable, and compliant barrier between the amputee's skin and the more rigid, weight bearing portions of the prosthetic socket [6].

Recently researchers have developed a variety of thicker gel materials that add a measure of cushioning and pressure dissipation while retaining the benefits of the original liners. Carbon fiber composites, developed by the aerospace industry, are increasingly being used in the artificial limbs, largely because of their superior strength to weight characteristics. There are currently many different design solutions of the above-knee prosthesis, by which researches are trying to enable and make an easier walking for limb amputees. It is attempted to make aboveknee prosthesis movable end enable its easier use. Large attention is directed towards finding the best construction of mechanical joint of knee and ankle. Helped by suitable medical therapeutics and exercises, amputees learn how to walk using their prosthesis. An important example is given by the so-called sports prosthesis which gives maximum aid to the sport amputees in achieving firstclass results [7].

The first artificial knee with an "on-board" computer to improve the symmetry of amputee's gait across a wide range of walking speed was developed by Blatchford in the early 1990s. Studies have confirmed that these "intelligent prosthesis" offer amputees a more reliable gait pattern during the swing phase of the gait cycle, permitting them to walk with more confidence and in a more energy efficient manner [8]. The Otto Bock C-Leg takes this a stage further, offering not only symmetry in the swing phase but also an improved security in the stance phase. Sensors in the ankle and shin of the prosthesis continually assess the position of the leg in space as the amputee is walking. The data are fed into two microprocessors inside the knee, and the resistance from a hydraulic damper is adjusted up to 50 times a second, optimizing both the stiffness throughout the entire gait cycle [9], as well as hydraulic damping, making both walking and stair descent easier [10].

However, without the usage of an outside power source, it is impossible for the body to perform several everyday activities which involve climbing significant slopes. This makes radically different solutions necessary [11].

Despite significant development of new technologies during the last decade, commercial below knee and above knee prostheses are still energetically passive devices. However, many locomotive functions, like walking up stairs and slopes, need significant power in knee and ankle joints. In addition, it has been shown that belowand above-knee amputees use more metabolic energy during level walking compared to the normal people gait. The additional power needed for the previously mentioned activities must be achieved by means of external energy sources that should be integrated in the prosthetic design.

Because of the presumption that robust external power sources are needed, which would be unacceptable for users, current lower limb prostheses incorporate knee and ankle joints with different types of passive damping throughout the gait cycle. There are computer-controlled prostheses which adjust the movement of the prosthesis to any speed and remarkably decrease step deviations and amputee effort. The most modern generation of controlled prostheses with computer-controlled phases of standing and swinging are an important step forward, since they offer the closest proximity to natural walking. Users of these prostheses can walk freely and safely at different speeds, even when walking on rugged ground or downstairs, still they cannot climb stairs as non-amputees do. The reason for this problem lies in the absence of external source of energy, which would have power generation capabilities comparable to an actual limb and would provide the energy required to lift the body.

In the process of ascending stairs, during loading response and midstance phase, body is being lifted from one stair to another and only one leg touches the stair and lifts the body, while another is in swing phase (in the air). In this phase the entire load is on one leg, and the body is in such position that moments in the knee and ankle joints are very high and exceed body weight by several times. 
In the case of above knee amputation, the lack of muscles makes it impossible to produce these forces, and passive prostheses which only use stored energy, are unable to perform such activities. This means, that for the prosthesis to be able to perform high demanding power activities, it must be powered, primarily in its main joints - knee and ankle.

\section{Hydraulic power system of the prosthesis}

Our prosthesis is designed in a way to mimic main leg movements in the sagittal plane. Its key features are knee and ankle joints which are movable in sagittal plane and are externally powered (Figure 1). In Figure 1 a reversible hydraulic power generator scheme is given for one linear actuator (in either knee or ankle joint). Movements of knee and ankle enable obtaining required kinematics of the prosthesis and power system enables its dynamic features [12]. Powered knee enables overcoming large forces that occur during loading response. It has been experimentally proven that having only powered knee in the prosthesis design is not enough, since joint in the ankle is also very important in natural stair ascent [13]. Powered ankle joint enables dorsal and plantar flexion movement of the entire foot, which provides better stabilization of the knee and the entire prosthesis and provides power needed in push-off phase.

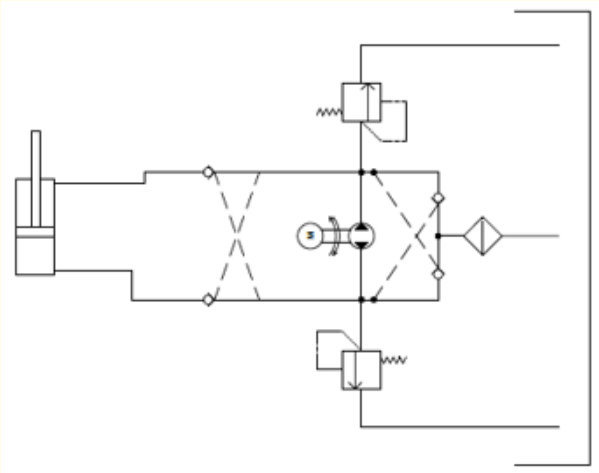

Figure 1: Hydraulic power generator scheme.

Our hydraulic power system concept is designed to enable characteristic movements of the prosthesis in sagittal plane while walking and ascending stairs. We are concentrated on testing the prosthesis on stair ascent, being the most complicated type of movement regarding energy requirements. The basic components of our hydraulic system are two hydraulic actuators, one for powering the knee and other for powering the ankle joint, hydraulic power pack unit and accompanying hydraulic installation (Figure 2 ). We chose off-the-shelf hydraulic power pack unit because it consists of all needed hydraulic installation components (electrical motor, hydraulic pump, reservoir, appropriate valves, connections etc.) integrated in one whole unit.

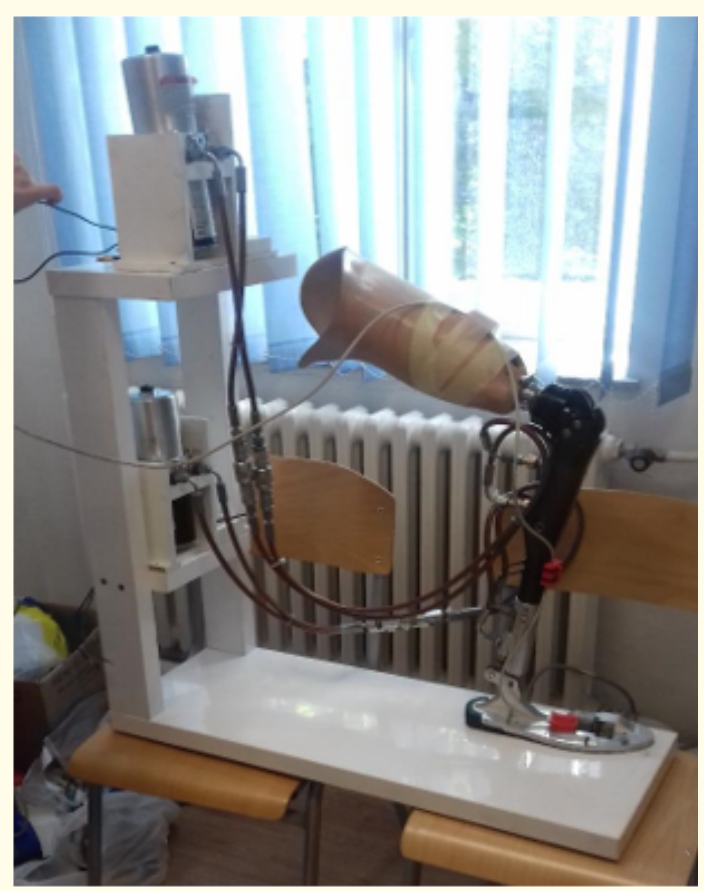

Figure 2: Separate hydraulic drive for knee and ankle joint.

\section{Prosthesis control design}

In the early days, a robot control system was considered as single input/single output linear system with each axis (joint) controlled independently. Coupling effects from the motion of other links were regarded as disturbances.

However, most robots are complex nonlinear and multivariable systems and uncertainty exists in the parameters defining manipulator dynamics. The dynamic equations of robot manipulators constitute complex, nonlinear and multivariable systems. One of the first methods of controlling these systems was inverse dynamics which is also known as a special case of the method of feedback linearization. It relies on cancellation of nonlinearities in the system dynamics. However, plant variability and uncertainty are obstacles to an exact dynamic inversion. Inverse dynamics control therefore has limited practical validity. To 
overcome this difficulty, motion control techniques based on the passivity property of the Euler-Lagrange equations are considered. Especially for the robust and adaptive control problems, the passivity-based approach shows significant advantages over the inverse dynamic method. Therefore, robust passivity-based control gained attention as a powerful nonlinear control law that can guarantee stability and tracking of arbitrary trajectories efficiently despite uncertainties in plant model parameters [14].

This project carries out modelling, control design and simulation tasks for a hydraulic knee and ankle joints in an organized way. The prosthetic knee and ankle are integrated into the mathematical model of the overall system. Open-loop control of the hydraulic knee (and similar design) has been considered before with optimization methods. To the extent of the author's knowledge, this is the first proposed robust feedback controller for this type of hydraulic knee and ankle system.

\section{Robust passivity-based controller}

Over the past decades, several control algorithms have been proposed for cooperative manipulators. The algorithms that can control both forces and motion can be divided into hybrid position/ force control schemes and impedance/admittance control schemes. In the hybrid control schemes, the coordination space is decoupled into motion and force-controlled directions, using a predefined and fixed selection matrix. Unexpected contact in motion-controlled directions can lead to damage of the object and manipulators, since the force in these directions is not controlled. In the impedance control schemes the dynamic relation between the forces and motion of the system is considered. Using impedance control, the task can be executed without leading to contact instability in the absence of precise knowledge of the contact directions. However, to achieve a satisfactory level of position/force tracking performance, the precise knowledge of contact directions is still required [15].

The controller proposed is of the cascade type: the prosthetic foot is regarded as an additional robotic link with direct torque control. The block diagram of this controller is shown in Figure 3. A motion controller is at the lowest level of this scheme. The inner motion control loop is added to improve the tracking performance. An online optimizer then finds the combination of cylinder piston positions that minimizes the difference between demanded moment and actual moment.

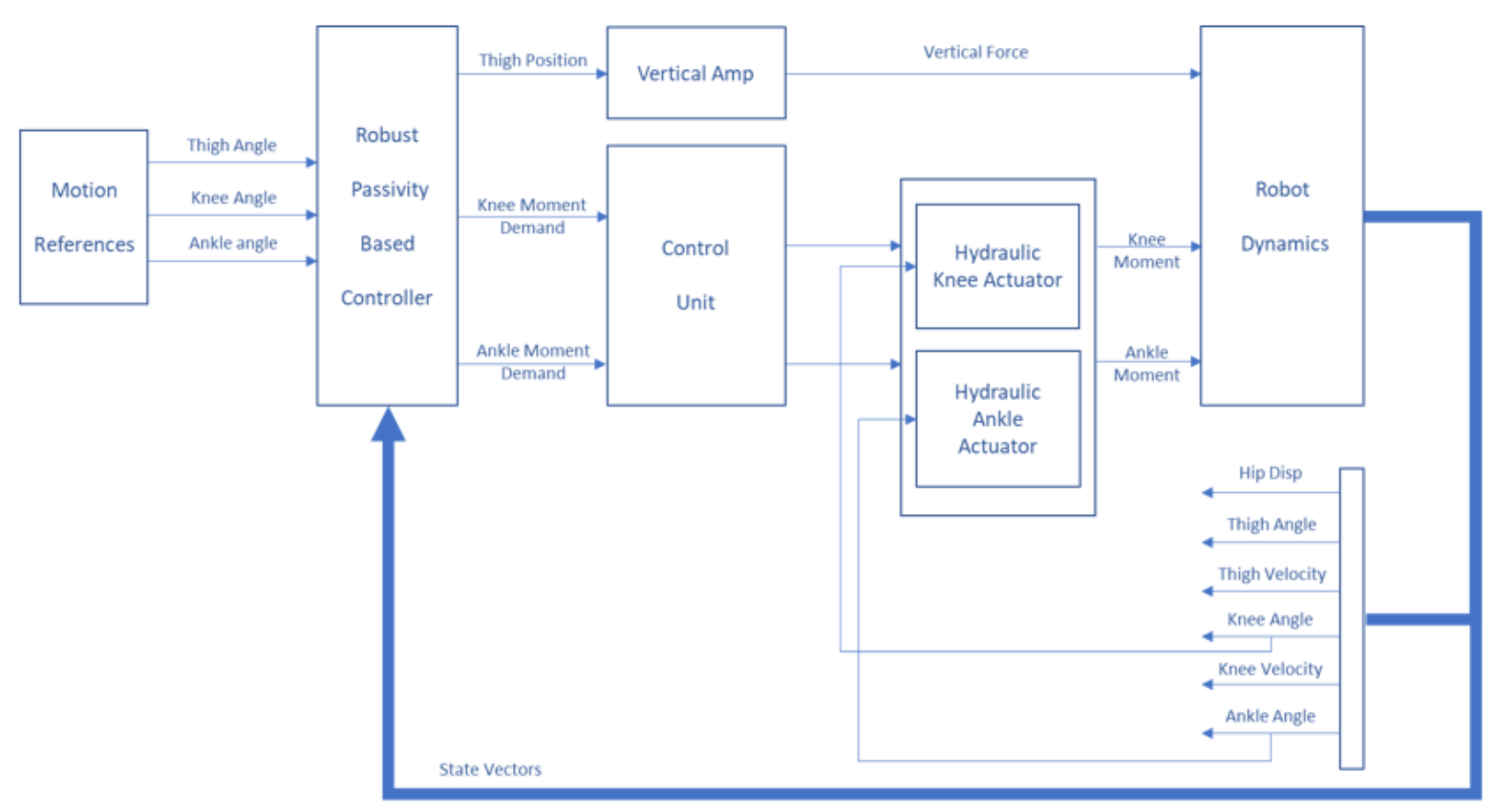

Figure 3: Cascade control architecture. 


\section{Control unit}

Control unit uses the developed control algorithm to control the joints of the above-knee prosthetic leg separately in order to follow the desired angle trajectories (Figure 4). Control sensors form an integral part of the control unit and allow the reference tracking of the prosthesis (Figure 5).

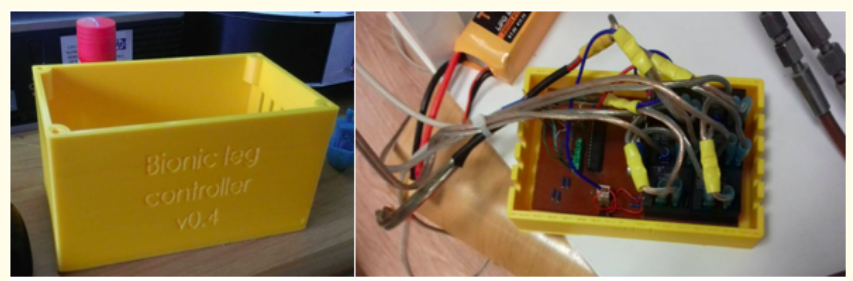

Figure 4: Control unit of the prosthetic leg.

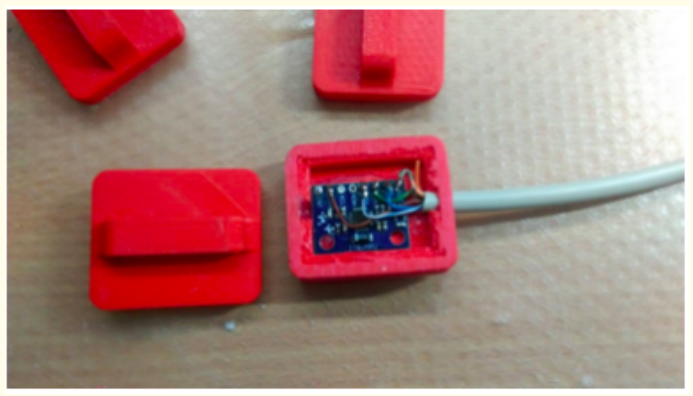

Figure 5: Control/measurement sensors.

For the measurement we used the sensors of our control unit, based on accelerometer and gyroscope. A set of three sensors is placed on the prosthetic leg prototype and can directly measure the angle of foot (metatarsophalangeal joint) and the angle in ankle and knee joints. The angle in the hip joint, here also referred to as thigh angle, is measured as the angle between the vertical axis and the position of the sensor mounted on the socket of the prosthetic leg.

In this version, for the first time, six sensors, which are a combination of a gyroscope and an accelerometer, are integrated along with the MPU 60/50 development board (Figure 6). Three sensors are placed on the prosthesis and three on the sound subject or the sound leg of an amputee. The sensors serve to map the angles of the leg and more precisely the angles of the knee, ankle and the foot. To facilitate the placement of sensors on the prosthesis and on the leg of a healthy subject, plastic cages were made for each sensor individually through $3 \mathrm{D}$ prototyping. The same is done for the electronics.

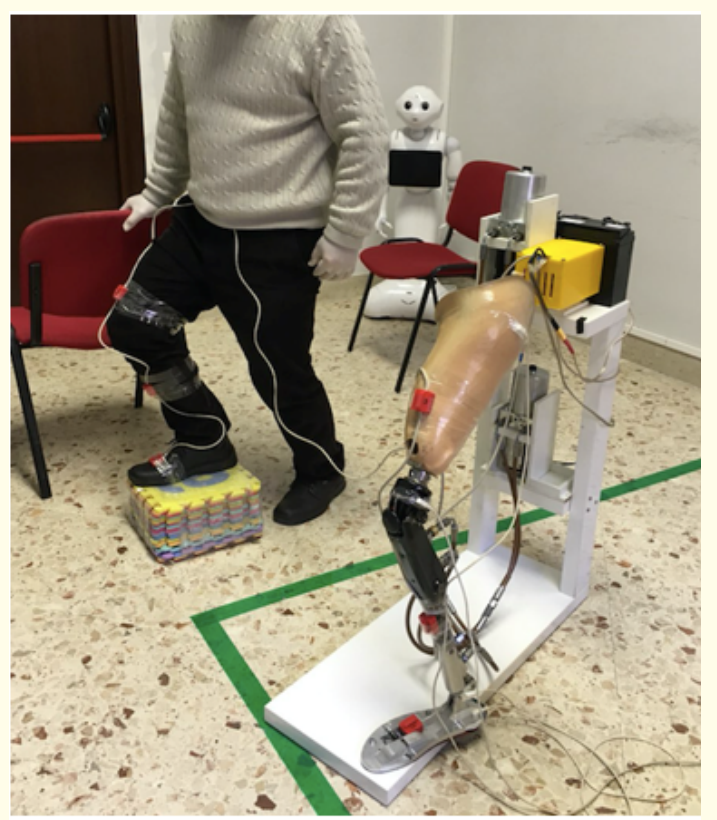

Figure 6: Smart Leg prototype in real time control testing.

The prosthetic controller determines the joint inputs required at each instant in time to make the prosthesis track a commanded motion [14]. This could be a sequence of end-effectors' positions and orientations or a continuous path. Various control methodologies are available for this kind of control, including proportionalintegral-derivative (PID) control, passivity-based control, robust control, adaptive control, etc. The control method used may cause significantly different performance. Therefore, each method has its own range of applications. For example, PID control, the most common type of control algorithm in industry, works well for independent joint setpoint regulation problems [15].

Robust passivity-based control for active above-knee prosthesis

For the purposes of simulation, the system of the active aboveknee prosthesis can be considered as a robotic manipulator consisting of three links with two one-axis joints. The coordinate system is set according to the standard Denavit-Hartenberg 
convention. The coordinate $\mathrm{q}_{1}$ represents the angle in the hip in relation to the vertical axis. Coordinates $\mathrm{q}_{2}$ and $\mathrm{q}_{3}$ represent the angles in the knee and ankle joint, respectively (Figure 7). In Figure 7 , the prosthetic part is shown in black, the remaining part of the human leg, including the hip and the point mass representing the rest of the healthy body is shown in grey. This model has been used in the simulations.

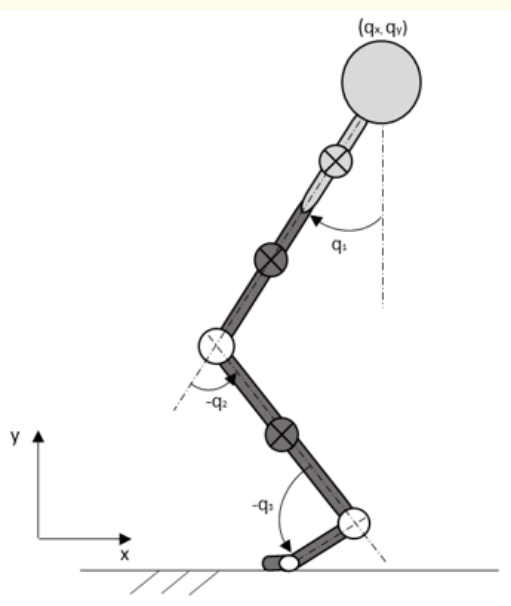

Figure 7: Model of active above-knee prosthesis [12].

Above-knee controller of the prosthetic leg receives input information for the knee and ankle joints $S_{i n}=\left\{q_{k}^{d}, q_{a}^{d}\right\}$ from the healthy human leg. Using linear transformation, set of input values for the hip joint $S_{h}=\left\{q_{h}, q_{h}^{d}\right\}$ is generated from $S_{i n}$, where $q_{h}^{d}$ is the desired path for $q_{h}$, the generalised coordinate representing the angle in the hip. Controllers use $S_{i n}$ to generate prosthetic knee moment during the swing phase and a period of stance, enabling the combined prosthetic system to mimic human movement, i.e $q_{a} \rightarrow q_{a}^{d} \Rightarrow q_{k} \rightarrow q_{k}^{d} \Rightarrow y_{h} \rightarrow y_{h}^{d}$ with limited tracking trajectory errors.

The flexible foot is attached to the ankle at an angle over the pylon. During the experiments, the foot will be placed on the Zebris plate [16] to measure the vertical force of the reaction of the ground (Figure 8). This data is implemented in the control algorithm as an external non-conservative force because it can play an important role in the feedback part of the control algorithm.

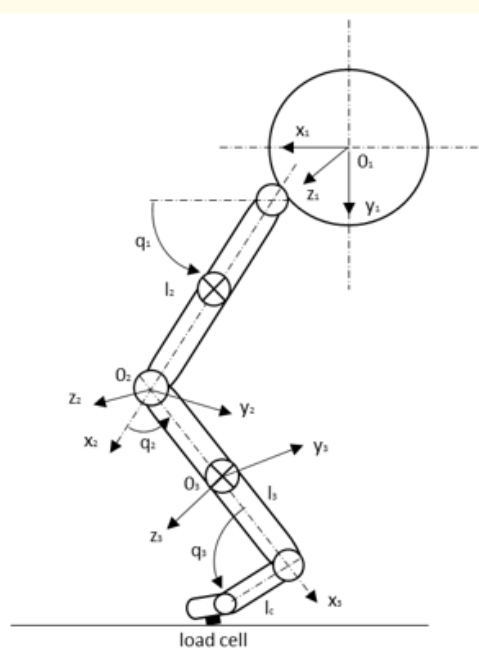

Figure 8: Coordinate plane.

In this case, the system can be considered as an active three-link planar robot, because the movement is only observed in the sagittal plane (Figure 8). A robotic dynamic model in joint coordinates can be written as:

$$
D(q) \ddot{q}+C(q, \dot{q}) \dot{q}+J_{e}^{T} F_{e}+g(q)=F_{a}
$$

where $q=\left[q_{1} q_{2} q_{3}\right]^{T}$ is the vector of joint angles, $D(q)$ is matrix of inertia, $\quad C(q, \dot{q})$ le vector of Centripetal and Coriolis torques, $J_{e}$ is the kinematic Jacobian of the point where external force acts, $g(q)$ is the gravitational vector and $F_{a}$ is the vector of combined actuator inputs, where the effects of inertia and friction are incorporated [14].

As the position vector of the point where the maximum value of the reaction force of the ground is known, its global location can be calculated using the transformation matrix:

$$
Z_{L C}=q_{1}-l_{c y} \cos \left(q_{2}+q_{3}\right)+\left(c_{3}+l_{c x}\right) \sin \left(q_{2}+q_{3}\right)+l_{2} \sin \left(q_{2}\right)
$$

where $l_{i}$ represents the length of the $i^{\text {th }}$ link of the mechanism; $l_{c i}$ represents the distance from the centre of the mass of the $i^{\text {th }}$ link to the previous joint.

Jacobian at the location of the maximum ground reaction force is given by: 
$J_{e}(1,1)=0$

$J_{e}(1,2)=-\left(c_{3}+l_{c x}\right) \sin \left(q_{2}+q_{3}\right)+l_{c y} \sin \left(q_{2}+q_{3}\right)-l_{2} \sin \left(q_{2}\right)$

$J_{e}(1,3)=-\left(c_{3}+l_{c x}\right) \sin \left(q_{2}+q_{3}\right)+l_{c y} \sin \left(q_{2}+q_{3}\right)$

$J_{e}(2,1)=J_{e}(2,2)=J_{e}(2,3)=0$

$J_{e}(3,1)=1$

$J_{e}(3,2)=\left(c_{3}+l_{c x}\right) \cos \left(q_{2}+q_{3}\right)+l_{c y} \sin \left(q_{2}+q_{3}\right)+l_{2} \cos \left(q_{2}\right)$

$J_{e}(3,3)=\left(c_{3}+l_{c x}\right) \cos \left(q_{2}+q_{3}\right)+l_{c y} \sin \left(q_{2}+q_{3}\right)$

Horizontal component of the foot velocity $V_{f}$ can be obtained from the Jacobian above, so the horizontal friction force $F_{G H}$ can be calculated as:

$$
F_{G H}=-\mu F_{G V} \operatorname{sign}\left(V_{f}\right)
$$

where $F_{G V}$ is the vertical component of the measured ground reaction force, $\mu$ is empirically calculated friction coefficient and equals 0.15 . Hence,

$$
F_{e}=\left[\begin{array}{lll}
F_{G H} & 0 & -F_{G V}
\end{array}\right]^{T}
$$

where $m_{1}$ is the mass of the upper part of the prosthetic leg and the corresponding components; $\mathrm{m}_{2}$ is the total mass of the lower part of the prosthetic leg and the associated components; $\mathrm{m}_{3}$ is the mass of the foot of the prosthetic leg; $\mathrm{m}_{0}$ is the equivalent inertial mass for rotational components belonging to the link 1 of the mechanism; $l_{2}$ is the nominal length of the upper part of the prosthetic leg; $l_{3}$ is the overall length of the lower part of the prosthetic leg, from the knee joint to the heel; $c_{2}$ is the distance from the centre of mass of link 2 to the origin of the coordinate system; $c_{3}$ is the distance from the knee to the centre of mass of link 3 including foot; $I_{2 z}$ is the total inertia of link 2; $I_{3 z}$ is the total inertia of link 3 including foot; $\mathrm{J}_{\mathrm{m}}$ is the inertia of the rotary engine; $f$ is the linear ratio of damping in link $1 ; b_{1}$ is the damping of the rotating actuator and $b_{2}$ is damping on the knee joint.

In order to run the three-link prosthetic leg robot with the robust passivity-based controller, the parameters of this manipulator are chosen as shown in Table 1 . In addition, the uncertainty level is determined as 0.1 in this simulation, which means the value of parameters is selected arbitrarily in the range of 10 percent fluctuation from the nominal value, the dead zone of the controller is chosen as 1 . The knee and ankle joints of the robot are driven by servo DC motors with amplifier gains $\mathrm{k}_{1}=375 \mathrm{Nm} / \mathrm{V}$ and $\mathrm{k}_{2}=15$ $\mathrm{Nm} / \mathrm{V}$. The hip joint, as a part of a remaining part of the body, for the sake of simulation is assumed to be ideally driven by torque directly.

\begin{tabular}{|c|c|c|}
\hline Parameters & Values & Units \\
\hline$m_{1}$ & 315.5 & $\mathrm{~kg}$ \\
\hline$m_{2}$ & 43.28 & $\mathrm{~kg}$ \\
\hline$m_{3}$ & 8.75 & $\mathrm{~kg}$ \\
\hline$m_{0}$ & 2.33 & $\mathrm{~kg}$ \\
\hline$l_{2}$ & 0.425 & $\mathrm{~m}$ \\
\hline$l_{3}$ & 0.527 & $\mathrm{~m}$ \\
\hline$c_{2}$ & -0.339 & $\mathrm{~m}$ \\
\hline$c_{3}$ & 0.32 & $\mathrm{~m}$ \\
\hline$I_{2 z}$ & 0.435 & $\mathrm{kgm}$ \\
\hline$I_{3 z}$ & 0.062 & $\mathrm{kgm}$ \\
\hline$J_{m}$ & 0.000182 & $\mathrm{kgm}^{2}$ \\
\hline$b_{1}$ & 9.75 & $\mathrm{Nms}$ \\
\hline$b_{2}$ & 1 & $\mathrm{Nms}$ \\
\hline$f$ & 83.33 & $\mathrm{Ns} / \mathrm{m}$ \\
\hline
\end{tabular}

Table 1: Simulation parameters for the three-link prosthetic leg.

\section{Results and Discussion}

In this section, simulation and measurement results are presented. Simulations show the implementation of the passivity based robust controller for the system of the active above-knee prosthesis with actuated knee and ankle joints. This controller is ideal for monitoring the change in the angles of the prosthetic leg with reference signals for a particular part of movement. The trajectory references for the hip, knee and ankle joints are normalized values of the corresponding angle trajectories of a healthy subject, as given in Figure 9. The controller is adjusted to give a better performance by tuning the controller gains $\mathrm{L}$ and $\mathrm{K}$ through trial and error.

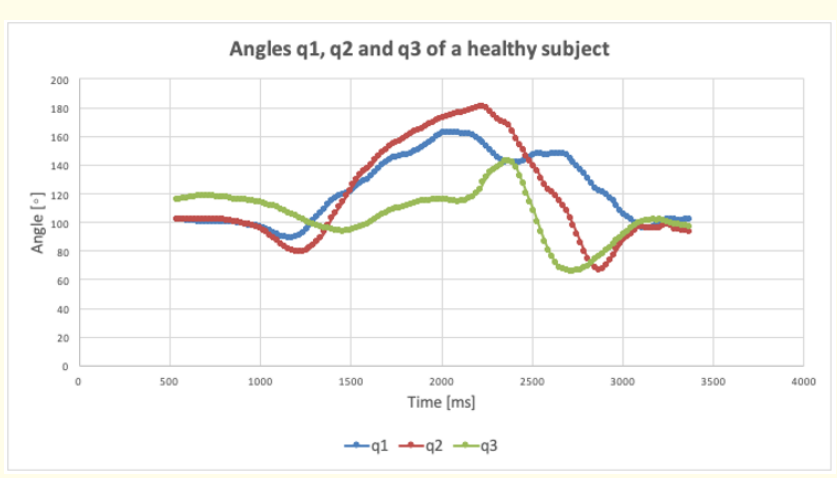

Figure 9: Reference signals of a healthy subject. 
The level of uncertainty is set to 0.1 , which means that $10 \%$ parametric uncertainty is present in the system. The angle of the hip joint is marked with $q_{1}$ and the angles in the knee and ankle joints with $q_{2}$ and $q_{3}$, respectively. Other simulation parameters are given in Table 1. It should be noted that the hydraulic aggregates of both activated joints of the prosthetic leg are driven by DC engines. In practice, the hydraulic power system is rather controlled through the voltage of the DC engines than via hydraulic valves. This control method proved to be effective in practice. However, in simulations the problem of control signal vibration is obvious. Although jumps in voltage signals are not too troublesome themselves, they can cause a problem in case of the hydraulic controller drive. The system is simulated for 20 seconds of which 5 seconds are shown in Figures 10-12. Diagrams show the tracking of the pre-set angular values in the knee and thigh joints. Measured output values are shown in red while desired reference input values are given in blue.

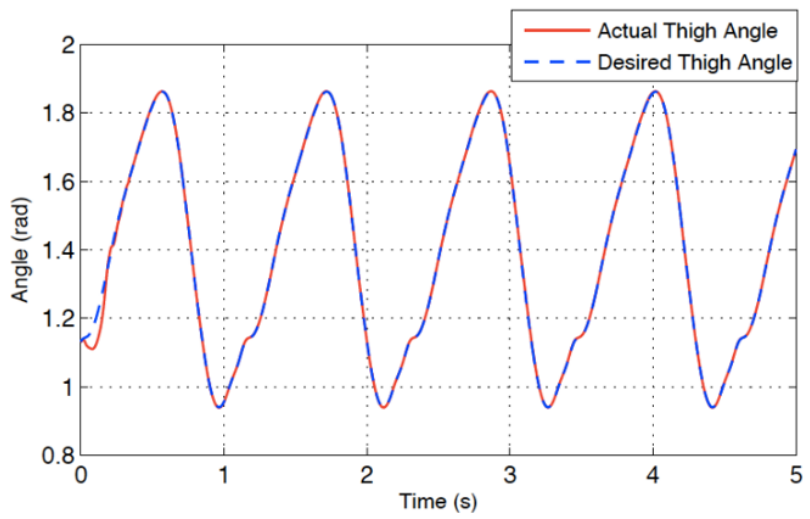

Figure 10: Comparison of thigh angle tracking between actual and desired value.

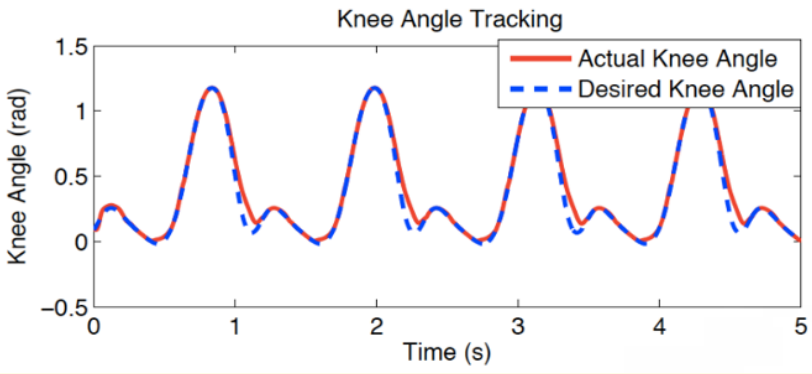

Figure 11: Comparison of knee angle tracking between actual and desired value.

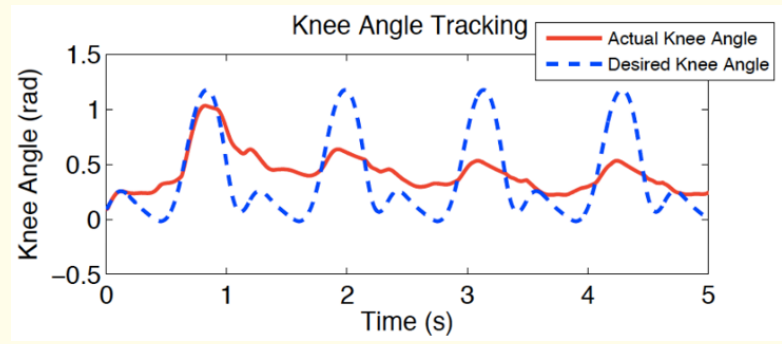

Figure 12: Knee angle tracking for hydraulic valve control.

If the management of the active hydraulic prosthesis is switched to the regulation of the flow through (servo) valves, then this mode of operation gives certain deviations. The deviations are the clearest in the knee angle where the greatest discrepancies can be seen (Figure 12).

In Figure 12 we see that the given values cannot be reached within the given time interval, which is set to $5 \mathrm{~s}$ in accordance with the experimental results. The reason is that large jumps at the controller input signals $u_{1}$ and $u_{2}$ negatively affect hydraulic system and can lead to wear and damage over time (Figure 13,14).

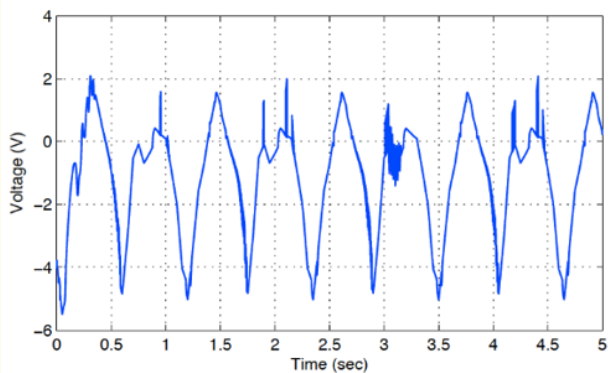

Figure 13: Control signal u1.

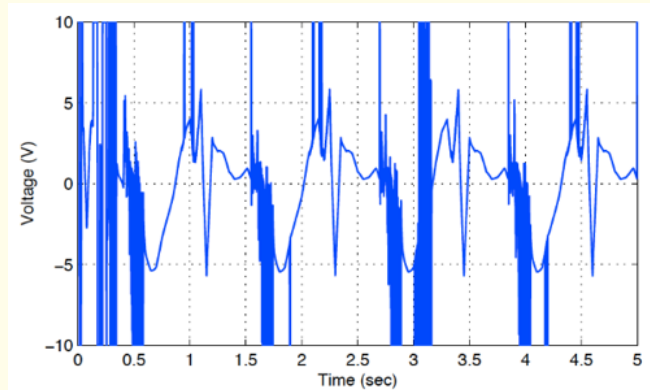

Figure 14: Control signal u2. 
By installing a low-pass filter this effect is significantly reduced and the results come in the range of what we saw in the regulation of the voltage of the DC motors of hydraulic aggregates (Figure 1517).

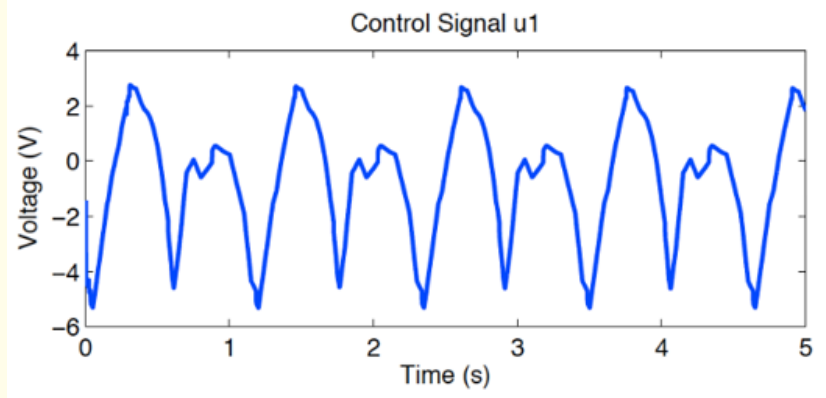

Figure 15: Control signal u1 after low-pass filter.

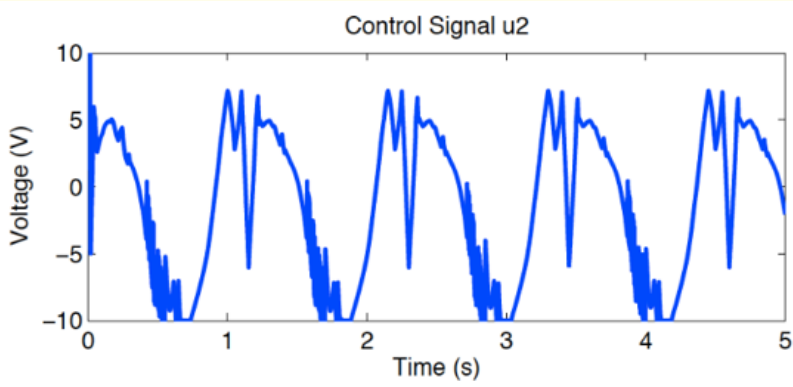

Figure 16: Control signal u2 after low-pass filter.

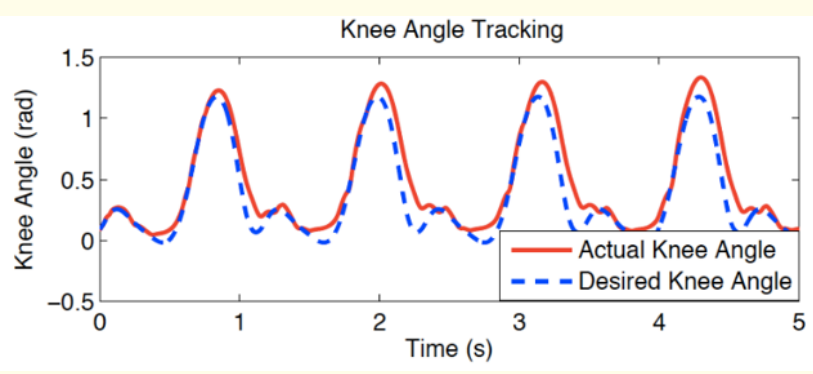

Figure 17: Knee angle tracking after implementation of low-pass filter.
The results of the simulations show that the passivity-based robust regulator is able to trace the trajectory of the joints close to the desired values, although another $10 \%$ of the parametric uncertainty is present within the model. Parametric uncertainty, which is an inevitable element in the robot management system, is successfully solved by the implementation of the robust passivitybased control.

\section{Conclusion}

The goal of the SmartLeg project is to prove a real possibility that a person with an above or below knee amputation can perform different types of motions which require power in knee and ankle joints, like climbing stairs, with hydraulic units whose weight and overall dimensions would not interfere with the comfort of the prosthesis user. SmartLeg prosthesis has the capability of active movement control in knee and ankle joints, which enable achieving necessary flexion and extension, as well as driving of below knee and above knee components. Developed wearable sensor network implements the proposed control in the prototype design, making it a 'smart prosthesis' and offers real time control.

In this paper we have presented preliminary investigations towards an active robotic prosthesis that could potentially enable people with an above- or below-knee amputation to perform different types of motions that require power in lower limb joints such as slope walking or stairs climbing. Our initial prototype, called SmartLeg, combines advanced mechanical design with adaptive control solutions. While the former aims at maximizing the functionality of the prosthesis form the engineering point of view (power consumption, stability, etc.), the latter is rather concerned with its usage.

The adoption of artificial intelligence and machine learning algorithms capable of learning and reproducing optimal motor patterns is in works and expected to provide an increased comfort for the end user and further customize the device to its particular needs.

Another proposed improvement is related to the optimization of the prototype mechanical design where the external power source will be integrated in the prosthetic housing. To be able to do it, the hydraulic power unit needs to be redesigned and custom made and some parts of the housing need to be redesigned as well. 


\section{Bibliography}

1. Hsu M-J., et al. "Psihological measurement of walking and running in people with transtibial amputations with 3 different prostheses". Journal of Orthopaedic and Sports Physical Therapy29.9 (1999): 526-533.

2. Marks L and Michael J. "Science, medicine, and the future: Artificial limbs.". British Medical Journal 323 (2001): 732-735.

3. Rosenfeld C., "Landmines - the human cost". ADF Health 1 (2000).

4. Meade $P$ and Mirocha J. "Civilian landmine injuries in Sri Lanka". Journal of Trauma 48.4 (2000): 735-739.

5. Islinger B., et al. "A review of orthopedic injuries in the three recent US military conflict". Military Medicine165.5 (2000): 463-465.

6. Heim M., et al. "Silicone suspension of external prosthetics; a new era in artificial limb usage". Journal of Bone and Joint Surgery 79.4 (1997): 638-640.

7. ZahediS. "The results of the field trial of the Endolite Intelligent Prosthesis, XII International Congress of INTERBOR (1993).

8. Buckley G., et al. "Energy cost of walking; comparison of "intelligent prosthesis" with conventional mechanism". Archives of Physical Medicine and Rehabilitation 78.3 (1997): 330-333.

9. Kastner J., et al. "What are the benefits of the Otto Bock C-Leg? A comparative gait analysis of C-Leg, 3R45 and 3R80". Techniques in Orthopaedics (1990).

10. Dietl H., et al. "C-Leg-un nuevo sistema para la protetizacion de amputados femorales". Orthopadie-Technik 3 (1998).

11. Zlatnik D. "Intelligently controlled above-knee prosthesis, Proc". IV International Conf". Motion and Vibration - MOVIC '98, Zurich, (1998).

12. Jelačić, Z. "Contribution to dynamic modeling and control of rehabilitation robotics through development of active hydraulic above-knee prosthesis, Doctoral dissertation, Faculty of Mechanical Engineering, University of Sarajevo, Sarajevo, Bosnia and Herzegovina (2018).
13. Dedić R., et al. Experimental investigation of the kinematics of above-knee prosthesis with hydraulic cylinders built in the knee and the ankle, 9th International Research/Expert Conference Trends in the Development of Machinery and Associated Technology TMT 2005, Antalya, Turkey, 26-30 September, (2005).

14. Jelačić Z., et al. Matlab Simulation of Robust Control for Active Above-Knee Prosthetic Device. Book title: Advanced Technologies, Systems, and Applications III. Lecture Notes in Networks and Systems, ISBN 978-3-030-02576-2, Publisher: Springer International Publishing AG, Copyright year: (2019).

15. Jelačić Z. "Impedance control in the rehabilitation robotics, Book title: Advanced Technologies, Systems and Applications II, ISBN 978-3-319-71320-5, Publisher: Springer International Publishing AG, Copyright year: (2018).

16. ZEBRIS measuring equipment.

17. Jelačić Z and Dedić R. "Real Time Control of Above-Knee Prosthesis with Powered Knee and Ankle Joints Book title: New Technologies, Development and Application II. Lecture Notes in Networks and Systems, ISBN 978-3-030-18072-0, Publisher: Springer International Publishing AG, Copyright year: (2020).

\section{Volume 2 Issue 7 July 2019}

(C) All rights are reserved by Zlata Jelačić. 